\title{
Influence of Deposition Potential on Structure of ZnO Nanowires Synthesized in Track-Etched Membranes
}

\author{
Alina Prună,,${ }^{a, b, *, d, z}$ Daniele Pullini, ${ }^{a}$ and David Busquets Mataix ${ }^{c}$ \\ ${ }^{a}$ Fiat Research Centre, Turin 10043, Italy \\ ${ }^{b}$ University Politehnica of Bucharest, Bucharest 010737, Romania \\ ${ }^{c}$ Departamento de Ingeniería Mecánica y de Materiales, Universidad Politécnica de Valencia, \\ Valencia 46022, Spain
}

\begin{abstract}
Single-crystal $\mathrm{ZnO}$ nanowires long up to several microns were fabricated by one-step electrochemical deposition. A template-based process employing track-etched polycarbonate (TE-PC) membranes was used for this purpose. The morphology and the structure characteristics of the $\mathrm{ZnO}$ nanowires were analyzed by means of Scanning Electron Microscopy (SEM), Focused Ion Beam (FIB), Transmission Electron Microscopy (TEM), and Selected Area Electron Diffraction (SAED). The growth process conditions turned out to have a marked influence on the crystal nature and morphology of the nanowires. Deposition rates ranging from $0.4 \mathrm{~nm} \mathrm{~s}{ }^{-1}$ and up to $0.6 \mathrm{~nm} \mathrm{~s}^{-1}$ were recorded for the growth of $\mathrm{ZnO}$ nanowires. The obtained results showed that by using carefully controlled deposition conditions single crystalline nanowires and fine-grained structures can be routinely obtained.

(C) 2012 The Electrochemical Society. [DOI: 10.1149/2.003205jes] All rights reserved.
\end{abstract}

Manuscript submitted November 9, 2011; revised manuscript received January 4, 2012. Published February 10, 2012.

$\mathrm{ZnO}$ has extrusive physical properties such as a direct bandgap in the ultraviolet range $(3.37 \mathrm{eV})$ or a large exciton binding energy $(60 \mathrm{meV})$ which have made it a great application prospect. ${ }^{1,2} \mathrm{ZnO}$ nano-objects have been widely investigated in the recent years owing to promising applications in nanodevices. They showed high sensitivity to different chemicals, have piezo- and pyro-electric properties, etc. ${ }^{3,4}$ As it has been shown that band edge, exciton energies, and UV luminescence of $\mathrm{ZnO}$ nanostructures are affected by crystallite size and crystal morphology, ${ }^{5,6}$ it is desirable to tailor their electronic properties in order to exploit the broad range of applications.

Nowadays, many efforts still focus on synthesizing oriented onedimensional $\mathrm{ZnO}$ nanostructures. Up to now, techniques using sophisticated and expensive equipments but also low temperature and cost-effective methods such as electrodeposition (ED) and hydrothermal deposition were employed for the synthesis of nanostructures. ${ }^{7-9}$ The major advantage of the electrochemical synthesis is given by its application for large area and high throughput productions, therefore suitable for an industrial use. ${ }^{10-13}$ The ED of ZnO nanowires has been focused mostly on the preparation of nanorod/nanowire arrays by the template method and on flat substrates. ${ }^{14,15}$

The $\mathrm{ZnO}$ electrodeposition is based on the reduction of an oxygen precursor such as dissolved molecular oxygen, nitrate ions or hydrogen peroxide. For example, Cembrero et al. ${ }^{16}$ reported on the cathodic electrodeposition of $\mathrm{ZnO}$ nanocolumns and nanowires from zinc chloride solutions saturated in molecular oxygen. The general scheme for $\mathrm{ED}$ of $\mathrm{ZnO}$ employing different oxygen precursors is supposed as follows.

The reaction at the cathode surface employing different oxygen precursors:

$$
\begin{array}{r}
\text { Nitrate: } \mathrm{NO}_{3}^{-}+\mathrm{H}_{2} \mathrm{O}+2 \mathrm{e}^{-} \rightarrow \mathrm{NO}_{2}^{-}+2 \mathrm{OH}^{-} \\
\text {Hydrogen peroxide: } \mathrm{H}_{2} \mathrm{O}_{2}+2 \mathrm{e}^{-} \rightarrow 2 \mathrm{OH}^{-} \\
\text {Molecular oxygen: } \mathrm{O}_{2}+2 \mathrm{H}_{2} \mathrm{O}+4 \mathrm{e}^{-} \rightarrow 4 \mathrm{OH}^{-}
\end{array}
$$

After hydroxyl anions are generated, they immediately precipitate in presence of $\mathrm{Zn}^{2+}$ to form $\mathrm{Zn}(\mathrm{OH})_{2}$ which is easily dehydrated above $65^{\circ} \mathrm{C}$ and forms $\mathrm{ZnO},{ }^{17}$ as presented in equations 4 and 5:

Chemical precipitation: $\mathrm{Zn}^{2+}+2 \mathrm{OH}^{-} \rightarrow \mathrm{Zn}(\mathrm{OH})_{2}$

\footnotetext{
* Electrochemical Society Active Member.

${ }^{\mathrm{d}}$ Present address: Institute of Materials Technology, University Politécnica of Valencia, Valencia 46022, Spain.

zz-mail: apruna@itm.upv.es; ai.pruna@gmail.com
}

$$
\text { Dehydration: } \mathrm{Zn}(\mathrm{OH})_{2} \rightarrow \mathrm{ZnO}+\mathrm{H}_{2} \mathrm{O}
$$

The process evolves itself drifting the $\mathrm{pH}$ of the electrolyte to a constant increase and to a local supersaturation of the bath in the vicinity of the electrode, thus provoking the precipitation on the electrode surface of $\mathrm{ZnO} ;{ }^{18}$ therefore, if a specific crystal order is desired, the $\mathrm{pH}$ of the electrolytic solution has to be precisely controlled and adjusted to a proper value.

As we know, control of morphology and structure (especially columnar structures) of oxides is of great importance for their implementation in technological devices. ${ }^{19,20}$ The templated ED technique offers a possibility to achieve these. However, even though the electrodeposition of $\mathrm{ZnO}$ nanowires was extensively studied, there are only a few researches on the structure of $\mathrm{ZnO}$ nanowires electrodeposited in porous membranes. ${ }^{15,21}$

As the ability of tailoring the properties of $\mathrm{ZnO}$ nanowires by ED technique and proper control of electrochemical parameters has not been fully exploited yet, it is therefore, mandatory to master the fabrication process over these properties through either chemical ${ }^{22,23}$ or physical routes ${ }^{24}$ for turning the fundamental research achievements into tangible products.

Here, the synthesis and structure study of the single-crystal $\mathrm{ZnO}$ nanowires grown in track-etched polycarbonate templates (TE-PC) is investigated by potentiostatic mode. The aim of this work is twofold. Firstly, we try to control the synthesis of $\mathrm{ZnO}$ nanowires by adjusting the electrodeposition potential. Secondly, we investigate the properties of the deposited $\mathrm{ZnO}$ nanowires and discuss the observed modification of structure in light of changing deposition potential.

\section{Experimental}

Materials. - The chemicals were reagent grade and used as received (Carlo Erba). Distilled water was employed for the preparation of the solutions. All the experiments were performed in $0.1 \mathrm{M}$ aqueous $\mathrm{KCl}$ solutions containing $5 \mathrm{mM} \mathrm{ZnCl}_{2}$. Hydrogen peroxide (30\% $\mathrm{H}_{2} \mathrm{O}_{2}$ (Carlo Erba)) was added in a molar concentration of $5 \mathrm{mM}$ and the $\mathrm{pH}$ value was adjusted at $6.4( \pm 0.02)$. Hydrogen peroxide was used as an oxygen precursor in the present paper, as it does not give rise to undesired reaction products and it is highly soluble in water (up to $10 \mathrm{M}$ ).

High-quality TE-PC membranes (Université Catholique de Louvain) were used as template in this study. The characteristics of the TE-PC membranes were: $21 \mu \mathrm{m}$ thickness, $50 \mathrm{~nm}$ pore diameter and $4 \times 10^{9}$ pores per $\mathrm{cm}^{2}$ pore density. TE-PC membranes were particularly used due to easiness in dissolution and removal to free the nanowires for further use and characterization. The templates were 
fabricated by using high-energy ions to create nuclear track damages in the polymer foils and by further etching step of the material to have nanopores drilled therein.

Set up.- In order to ensure the electrical continuity, a thin gold layer ( $30 \mathrm{~nm}$ thick) was sputtered on one side of the TE-PC membrane using a BOC Edwards sputter coater; the membrane was then used as the working electrode (WE) in a three-electrode electrodeposition cell and placed in such a manner to expose the uncoated side of the template to the electrolyte. Pt wire was used as the counter electrode (CE) and saturated calomel (SCE) served as the reference electrode (RE). All the potential values were recorded with respect to the SCE.

Synthesis methods. - All electrochemical measurements were performed on an AMEL 500 potentiostat. The temperature of the electrolytic bath was set to $75^{\circ} \mathrm{C}$, unless stated otherwise. Prior to the experiments, the WE was sonicated in the electrolyte for 10 minutes to promote its infiltration within the template nanopores. Moreover, 30 minutes of immersion in the electrolyte was allowed to WE in order to ensure the temperature is constant throughout the TE-PC template. Omitting this step will result in the growth in some pores first. Moreover, the number of pores in which growth proceeds with time may be changing. In order to take a first glance at the ED process, cyclic voltammetry studies were carried out in order to determine the deposition potential window. The cell voltage was swept from open circuit potential to $-1.5 \mathrm{~V}$ at a sweep rate of $50 \mathrm{mV} \mathrm{s}^{-1}$. The $\mathrm{ED}$ of $\mathrm{ZnO}$ in the TE-PC templates was carried out by using the potentiostatic mode employing values in the range -0.95 to $-1.3 \mathrm{~V}$ for different growth time. The growth was interrupted before the transition to bulk film in order to easily release the nanowires upon removal of the template.

Characterization methods. - Growth of nanowires was achieved by analyzing the chronoamperometric curves recorded for each deposition potential employed. Scanning Electron Microscopy (SEM) and Focused Ion Beam (FIB) images of the $\mathrm{ZnO}$ nanowires were obtained by using a FEI Quanta 3D 200 dual beam microscope at an acceleration voltage of $30 \mathrm{kV}$ following the partial dissolution of the template in dichloromethane and evaporation of solvent. Transmission Electron Microscopy (TEM) and Selected Area Electron Diffraction (SAED) were performed in order to analyze the effect of deposition potential on the crystalline structure of obtained nanowires. The $\mathrm{ZnO}$ nanowires were freed by dissolving the template membrane in dichloromethane. To have the nanowires ready for characterization, all samples were flushed several times, rinsed with ethanol, and finally dried in clean air. A Phillips CM-10 microscope working at $100 \mathrm{kV}$ was employed for these measurements.

\section{Results and Discussion}

Prior to the nanowires deposition, cyclic voltammetry studies were performed on $\mathrm{Au}$ covered TE-PC templates as reported in Figure 1a. The method consists in generating hydroxide ions at the surface of the cathode by reduction of the oxygen precursor. The onset of $\mathrm{ZnO}$ deposition was observed around $-0.75 \mathrm{~V}$ and the absence of any oxidation peak on the anodic curves that could lead to $\mathrm{Zn}$ formation was confirmed. Therefore, potential values more negative than $-0.7 \mathrm{~V}$ could be used to deposit $\mathrm{ZnO}$ structures. In the following, potential values in the range -0.95 to $-1.3 \mathrm{~V}$ were applied and effect of deposition potential on the obtained $\mathrm{ZnO}$ nanowires was assessed. As potential and current distribution is governed by phenomena related to charge transport in the electrodes and charge/mass transport in the electrolyte, ${ }^{25}$ the infuence of overpotential on current distribution must be taken into account.

Figure $1 \mathrm{~b}$ presents the chronoamperometric curve corresponding to the deposition of $\mathrm{ZnO}$ nanowires at the constant potential of $-1.1 \mathrm{~V}$. The current growth presents some variations due to the bubbles formed on the working-electrode surface. The reduction process takes place upon the displacement of the $\mathrm{Zn}^{2+}$ ions in the direction of the cathode when the system is driven out of electrostatic equilibrium

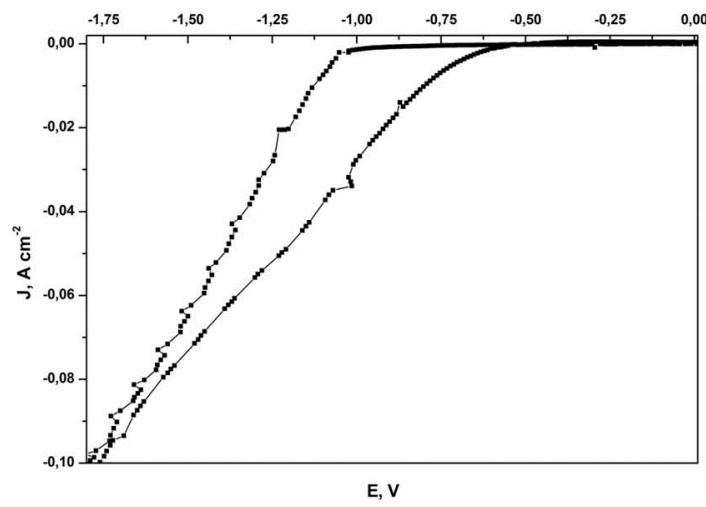

(a)

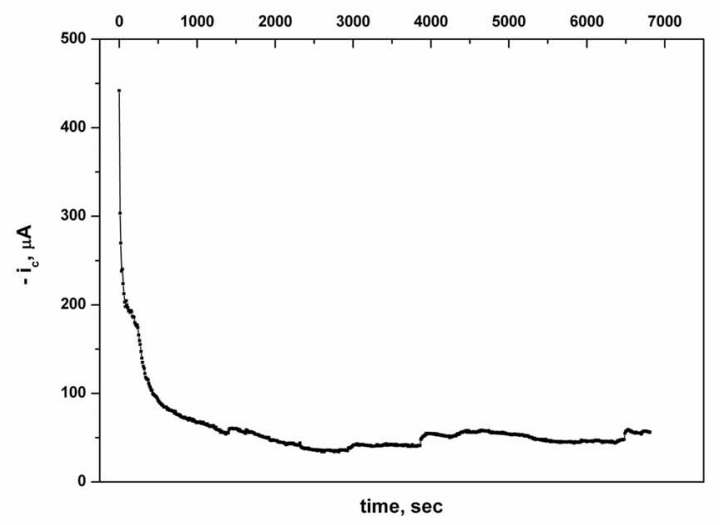

(b)

Figure 1. a) Voltammetric curve of Au covered TE-PC substrates in $0.1 \mathrm{M}$ $\mathrm{KCl}+5 \mathrm{mM} \mathrm{ZnCl}_{2}+5 \mathrm{mM} \mathrm{H}_{2} \mathrm{O}_{2}$ at a scan rate of $50 \mathrm{mV} \mathrm{s}^{-1}$ and b) Current transients recorded for the growth of $\mathrm{ZnO}$ nanowires at $-1.1 \mathrm{~V}$.

due to the electric field generated in the solution. After the initial transient caused by the induction process (the charge-discharge of the double layer) at the electrode/electrolyte interface, the current reaches a constant value as the cations in the close vicinity of the electrode are being reduced. This corresponds to the formation of $\mathrm{ZnO}$ nuclei. Further, the electrodeposition process is balanced while nanowires grow up. An explanation of I-t transient could be supported by applied voltage and, if mass transport is a limitation, by the diffusion of ions, which are generally known to determine the value of electrochemical current. Since the RE is located in the bulk electrolyte above the TE-PC membrane, there may be a voltage drop along the electrolyte in the pores which would result in a reduced electrochemical current. In case of progressive growth, this voltage drop should decrease since the wires are approaching the bulk and so, the current would increase. A similar conclusion is reached if mass transport limitation is considered. As a first-order approximation, one may assume a constant ion concentration in the electrolyte above the pores. The concentration gradient, which determines the diffusion current, increases if the wires approach the top surface of the membrane, so that the diffusion current increases as well. Apart from diffusion and migration, the electrochemical current is also proportional to the electrode area.

The growth rate of $\mathrm{ZnO}$ nanowires into the TE-PC templates is presented in Figure 2a as a function of the deposition potential for the same deposition duration. Since $\mathrm{ZnO}$ formation has generally a fast kinetics, the local composition of the electrolyte around the nanowires will be mainly determined by the ratio between the rate of $\mathrm{OH}^{-}$generation and the diffusion of $\mathrm{Zn}^{2+}$ ions to the cathode. As it is obvious, by increasing the overpotential, nanowires will grow faster in the pores and so, the filling time decreases while the filling rate increases. The growth rate of $\mathrm{ZnO}$ nanowires ranged $0.4-0.6 \mathrm{~nm} \mathrm{~s}^{-1}$ and, as it is ascertained from Figure 2a, the 


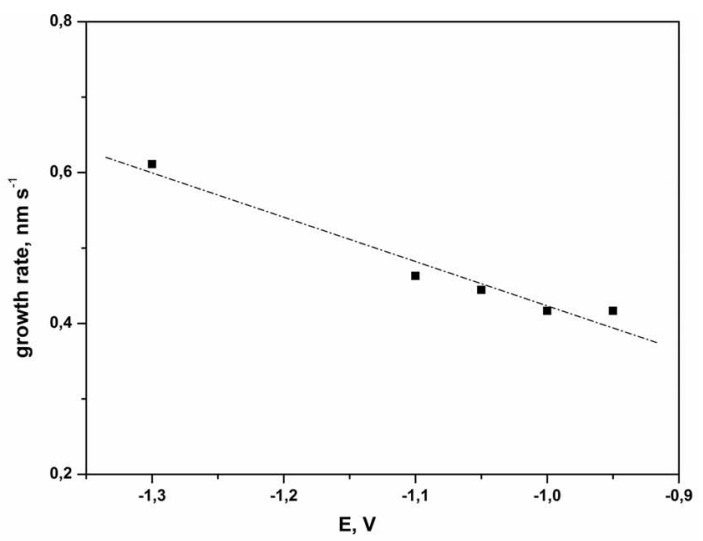

(a)

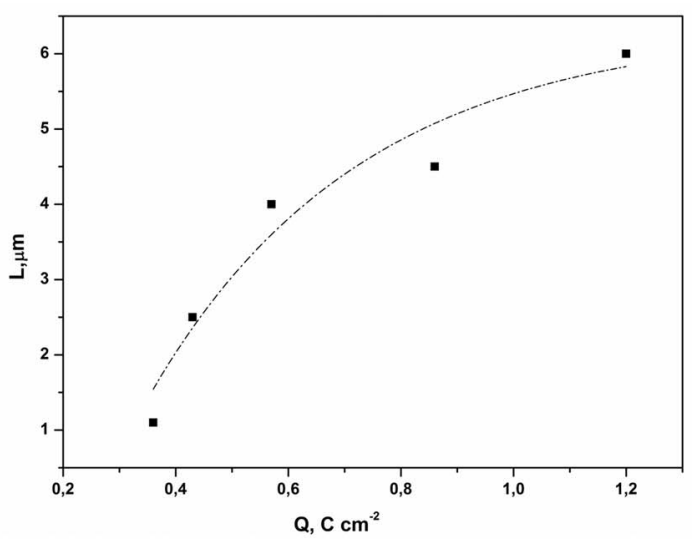

(b)

Figure 2. a) Growth rate of $\mathrm{ZnO}$ nanowires as a function of deposition potential ((--) is the fitted line), b) variation of nanowire length with electric charge ((o) is the fitted curve).

dependence on the deposition potential followed a linear trend. For example, 1- $\mu \mathrm{m}$-long $\mathrm{ZnO}$ nanowires could be deposited by electrochemical deposition only for approximately $40 \mathrm{~min}$ at the deposition potential of $-1.1 \mathrm{~V}$. Therefore, it is possible to control the aspect ratio of nanowires by changing the parameters such as the applied potential or deposition time.

The length of the nanowires increases with time as shown in Figure $2 b$ depicting the dependence of the nanowire length on the deposition charge, at a given deposition potential value. The theoretical electrical charge transferred was calculated by the formula:

$$
\mathrm{Q}_{\mathrm{th}}=\mathrm{zF} \rho \mathrm{A}_{\mathrm{eff}} \mathrm{L} / \mathrm{M}
$$

where $\mathrm{z}$ is the number of exchanged electrons, $\mathrm{F}$ is the Faraday constant, $\rho$ is the density of the deposited material, $\mathrm{L}$ is the length of the nanowires, $\mathrm{A}_{\text {eff }}$ represents the effective area of nanopores $\left(\mathrm{cm}^{2}\right)$ and $\mathrm{M}$ is the molecular mass of $\mathrm{ZnO}$. Here, a $100 \%$ current efficiency is assumed. The $A_{\text {eff }}$ value is given by:

$$
\mathrm{A}_{\text {eff }}=\mathrm{A}_{\exp } \pi \mathrm{r}^{2} \mathrm{~N}
$$

where $A_{\exp }$ is the exposed TE-PC area, $r$ is the nanowire diameter and $\mathrm{N}$ is the surface density of pores of the TE-PC membrane. For all the experiments carried out, the deposition efficiency was calculated from the chronoamperometric curve and the deposited mass by using the experimental and theoretical electrical charge values. As expected, the deposition efficiency was rather low - the average deposition efficiency calculated was close to $6 \%$ similar to what Elias et al. ${ }^{26}$ obtained while growing $\mathrm{ZnO}$ nanowires on planar ITO substrate. The presence of impurities and heterogeneities (grain boundaries) could have suppressed the nanowire nucleation. Moreover, the growth could be limited by the $\mathrm{OH}^{-}$at the top of the wire or by $\mathrm{Zn}(\mathrm{OH})_{\mathrm{x}}{ }^{2-\mathrm{x}}$, where $\mathrm{Zn}(\mathrm{OH})_{\mathrm{x}}{ }^{2-\mathrm{x}}$ could form at the electrode by the reaction of $\mathrm{Zn}^{2+}$ and $\mathrm{OH}^{-}$.

It is noted that the increase of nanowire length with electrical charge (i.e. time) follows an exponential law at a given overpotential. An explanation for this trend could be as follows: during the growth period of the nanowires, the precursor could continuously provide liberally reactants for electrodeposition by ion diffusion and the variation of $\mathrm{HO}^{-}$and $\mathrm{Zn}^{2+}$ concentrations could be negligible at the substrate surface in a certain period up to the necessary time (corresponding to a charge of $0.6 \mathrm{C} \mathrm{cm}^{-2}$, in our case) - see image (A) in Figure 3. Above this period of time, the solution facing the WE surface depletes from ions and so, the precursor could not supply adequate reactants for the growth of nanowires. A boundary layer is formed near the membrane surface-see image (B) in Figure 3. In this region, transport phenomena supply the electrode with ions coming from the solution and a diffusion controlled limiting current density would be obtained. ${ }^{27,28}$ In general, the boundary layer reduces the effectiveness of the entire process due to the area of oriented $\mathrm{H}_{2} \mathrm{O}$ molecules formed in front of the cathode. Therefore, the crucial steps become the diffusion process of ions and electrochemical reduction of $\mathrm{H}_{2} \mathrm{O}_{2}$, which will result in the decrease of the growth rate. The general scheme of the electrodeposition mechanism is presented in image (C) in Figure 3, based on the reactions 2,4 , and 5 .

One of the most common models used to interpret chronoamperometry data is the Cottrell equation. ${ }^{29}$ In case of a reduction reaction and before the occurrence of the limiting current, it can be written as:

$$
\mathrm{i}(\mathrm{t})=\left(n F A_{e f f} D^{1 / 2} \mathrm{C}\right) /\left(\pi^{1 / 2} \mathrm{t}^{1 / 2}\right)
$$

where $i(t)$ is the diffusion current, $n$ is the number of electrons transferred in one electrochemical reaction step, $\mathrm{F}$ is the Faraday constant, $\mathrm{A}_{\text {eff }}$ is the effective electrode area (exposed electrode area at the bottom of the pores given by the equation II), D is the diffusion coefficient of electroactive species in electrolyte, $\mathrm{C}$ is the initial bulk concentration of electroactive species in electrolyte and $t$ is the time.

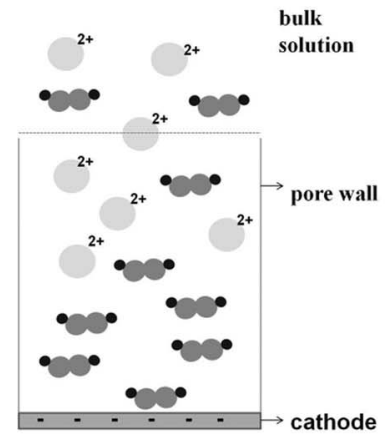

(A)

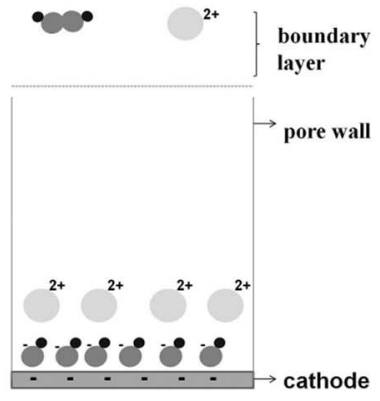

(B)

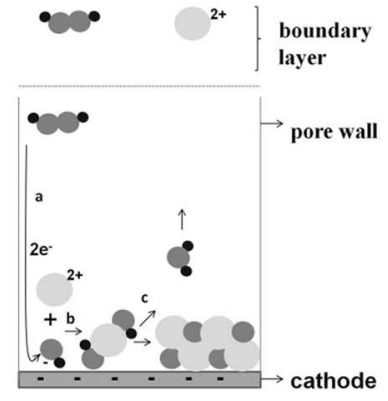

(C)
Figure 3. Schematic of the electrodeposition of $\mathrm{ZnO}$ from $\mathrm{ZnCl}_{2}$ solution with $\mathrm{H}_{2} \mathrm{O}_{2}$ as oxygen precursor. 


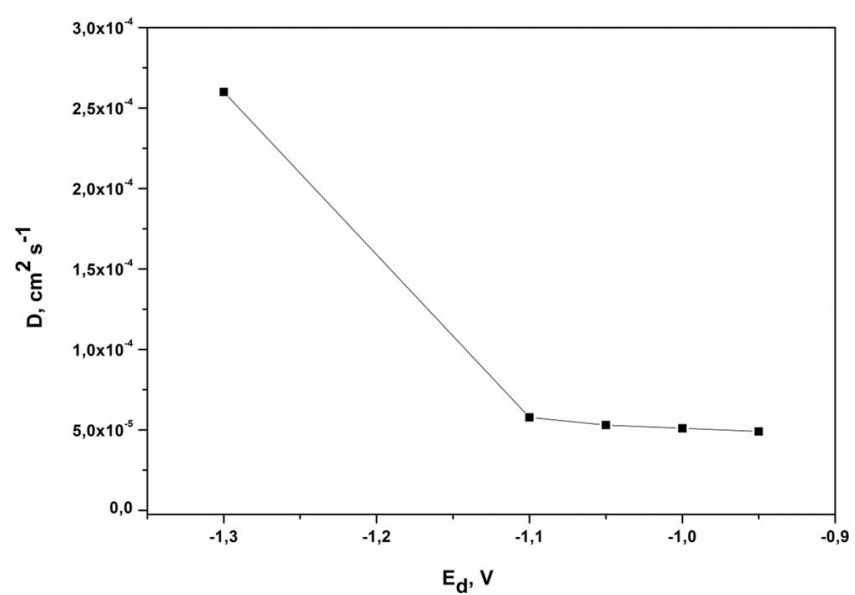

Figure 4. Calculated diffusion coefficient (D) at different deposition potential values.

The Cottrell equation was derived based on semi-infinite planar diffusion and assuming that the voltage step was sufficiently large so that the surface concentration of active species could be essentially zero. The diffusion coefficient can be extracted from the plot of $i(t)$ $=\mathrm{f}\left(\mathrm{t}^{-1 / 2}\right)$. In the present work, the obtained plots were not linear for which a number of possible explanations may be given. The first and most obvious explanation is that the boundary conditions used to derive equation III do not accurately represent the physical processes occurring in the electrode and there are surface and pores irregularities, making the characteristic length of the diffusion field much smaller. The appropriate time interval from which the Cottrell slope should be calculated can be found by plotting $\mathrm{I} \times \mathrm{t}^{1 / 2}$ versus $\mathrm{t}^{1 / 2} \cdot 3^{30}$ The results presented in the present work are obtained according to the methods described above.

Nevertheless, the Cottrell relation is not valid for longer times due to the formation of a radial instead of a planar diffusion field. In case of a spherical electrode, the Cottrell equation is as follows: ${ }^{29}$

$$
\mathrm{i}(\mathrm{t})=\left(\mathrm{nFA}_{\mathrm{eff}} \mathrm{D}^{1 / 2} \mathrm{C}\right) /\left(\pi^{1 / 2} \mathrm{t}^{1 / 2}\right)+\left(\mathrm{nFA}_{\mathrm{eff}} \mathrm{D}^{1 / 2} \mathrm{C}\right) /(\delta)
$$

where the time independent second term includes $\delta$ which is the radius of the sphere. To determine the effective diffusion coefficient during long times and to compare it with the Cottrell data, the radial diffusion equation IV could be applied.

The average values of diffusion coefficient, D (planar and radial), ranged from $4.9 \times 10^{-5}$ to $2.6 \times 10^{-4} \mathrm{~cm}^{2} \mathrm{~s}^{-1}$ as observed in Figure 4 presenting the influence of deposition potential on the diffusion coefficient value. Although these values are higher than reported values in literature for planar electrodes ${ }^{31}$ some explanation could arise from the electrodeposition conditions, for example the ultrasonication pre-treatment of the TE-PC and the time allowed to the TE-PC to reach and maintain the deposition temperature that could significantly improve the diffusion. Nevertheless, an accurate experimental determination near the electrode becomes difficult due to cell geometry and electrode dimensions. Information about the dimensions and geometry of the interpenetrating and spatially complementary solid and porous fractions is needed. The distribution of electrode material must also be taken into account. The main approximation would be to consider that the concentration at the top of a pore is constant, independent of the distance measured from the center of the pore to its edges. ${ }^{32}$ The next step would be to consider the relative dispositions of the liquid within it, that is, the capillary equilibrium. This demands a detailed description of the porous medium, not only in geometrical terms but also in terms of wetting characteristics, and also involves historical considerations, in that the particular order of electrode filling becomes significant. For the calculation of the electrochemical characteristics of porous electrodes, the description of the state of capillary equilibrium appears to be fundamental. However, the problems in experimentally determining of such equilibrium states are considerable. The most important hindrance to the mathematical description of porous media is due to difficulty in describing the irregular structures of real electrodes. This necessitates the use of probability theories in developing and testing different plausible models. The radius and wetting angle have a marked importance in characterizing a pore, as well. Moreover, if the sites of electron transfer are discrete, i.e. the electrode is partially blocked, the diffusion field close to the surface can be hemispherical or a combination of hemispherical and linear geometries. ${ }^{33}$ Diffusion through porous media should consider even more: tortuosity coefficient $\theta$ (the ratio of the mean increase in diffusional path per unit length in the direction of diffusion within the material) and pore entrance effects. ${ }^{34}$

It is well known that properties of all materials are determined by their structure. Even minor structural differences often have profound effects on their properties. ${ }^{35}$ Figure 5 shows the SEM

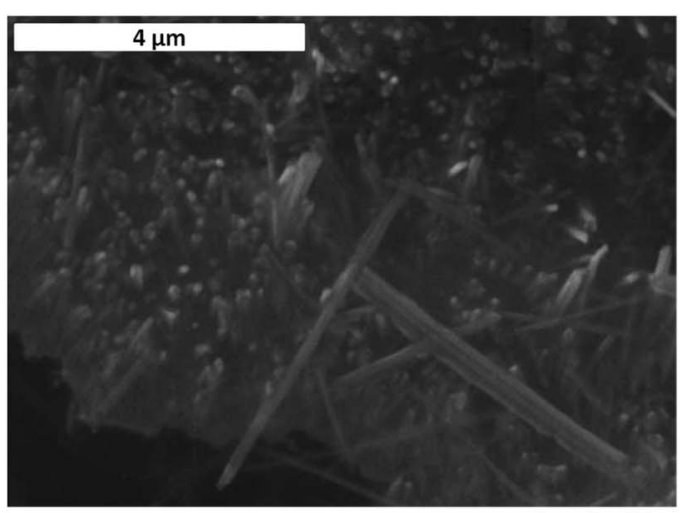

(a)

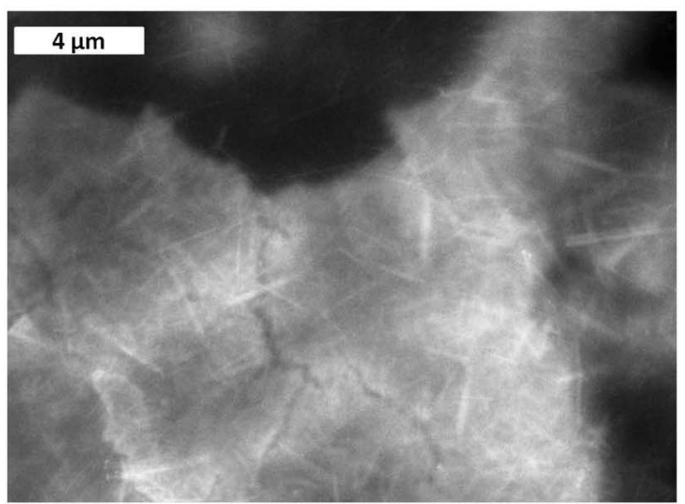

(b)

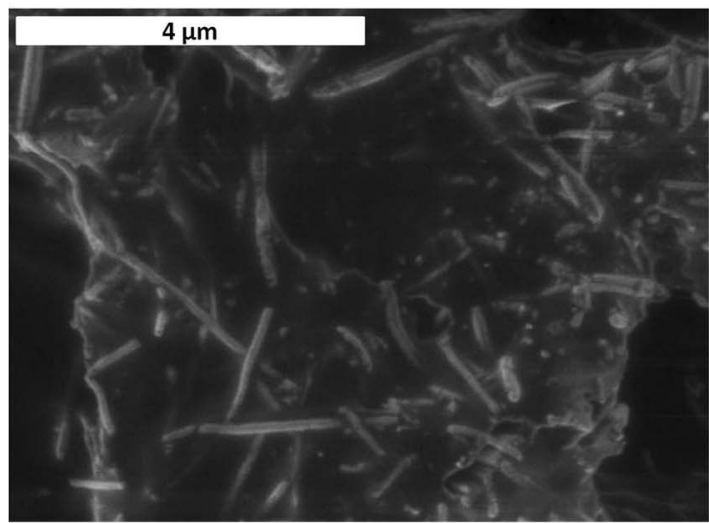

(c)

Figure 5. SEM-imaged $\mathrm{ZnO}$ nanowires obtained at: a) $-1.1 \mathrm{~V}$ and b) $-1.3 \mathrm{~V}$, after partial dissolution of membrane; c) FIB image of the same nanowires as in $5 b$. 


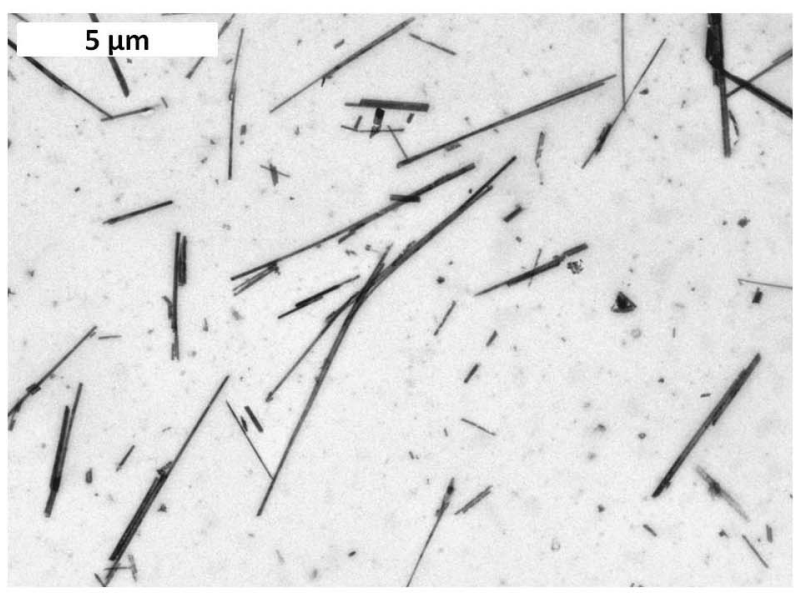

(a)

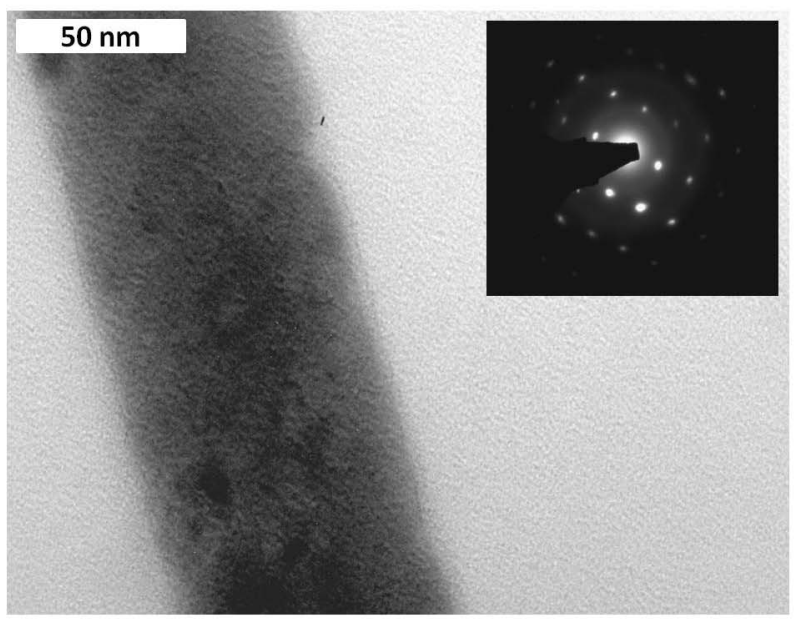

(c)

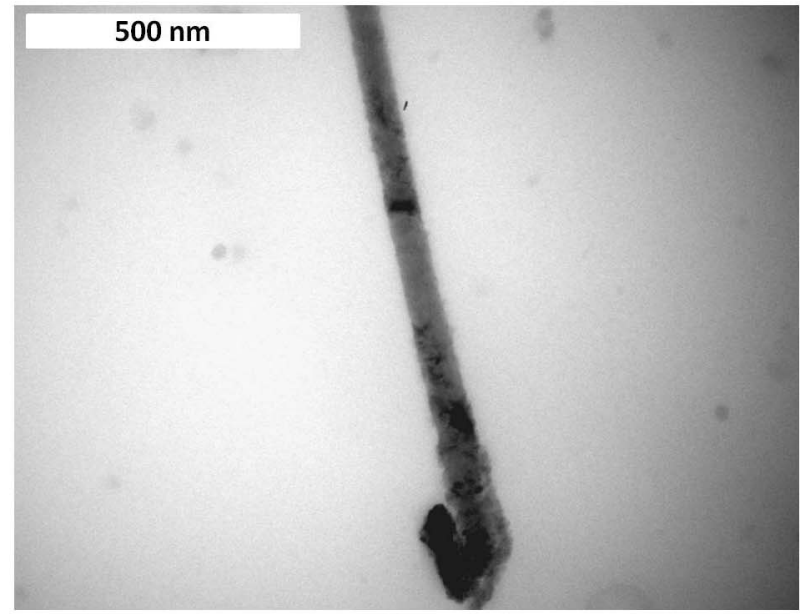

(b)

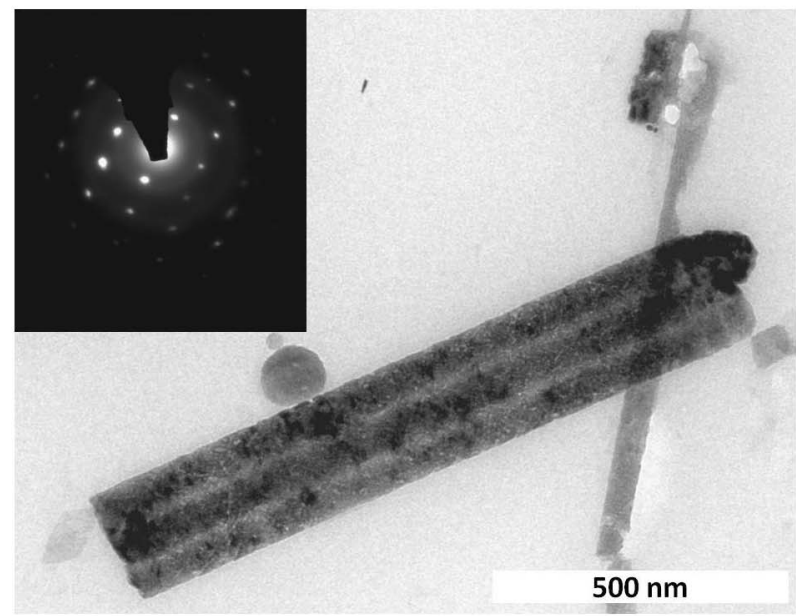

(d)

Figure 6. TEM images showing: a) length distribution, b) diameter, c) single-crystal structure and d) three portions of single-crystal ZnO nanowires electrodeposited at $-1.1 \mathrm{~V}$.

images confirming the formation of $\mathrm{ZnO}$ nanowires by potentiostatic deposition at $-1.1 \mathrm{~V}$ and $-1.3 \mathrm{~V}$. The nanowires presented in Figures $5 \mathrm{a}$ and $5 \mathrm{~b}$ are still partially embedded in the polymeric membrane and have a low contrast. By using FIB imaging - Figure 5c, the contrast was increased and resulted in a better defined image of the nanowires. In all these figures, the nanowires appear randomly distributed and mostly lying on the substrate. This is mainly due to the partial dissolution of the TE-PC template by solvent evaporation and the mechanical forces involved which also results in a random distribution in the nanowire length independently of deposition potentials employed. Another explanation for this variability comes from an intrinsic nature of TE-PC membranes. The pores in these membranes are not aligned parallel but also have a considerable angular distribution. For this reason, the length of the pores, measured through the membrane, is not constant. Hence, even if the electrochemical deposition would proceed at an exactly constant rate, the complete nanopore filling would still vary among different pores.

In order to have a more precise estimation of the diameter distribution of nanowires fabricated in the same conditions and to assess the effect of the deposition potential on the structure of the $\mathrm{ZnO}$ nanowires, TEM imaging was used. Figure 6 presents the TEM micrographs of the electrodeposited nanowires at $-1.1 \mathrm{~V}$. The overall TEM image in Figure 6a shows that the variability of nanowire length was higher than estimated and justified by membrane properties. The length of the imaged nanowires cannot be considered as representative of the arrays because a wide distribution was observed for nanowires from the same sample. The observed inhomogeneous growth could be explained by the non-uniform velocity in the pore array resulting in mixing of the fluid in the flow direction, which further results in a dispersion of the concentration profiles. In this case, there are some initial differences in the pore array (for instance, if some of them are initially poorly wetted) and the number of pores in which electrodeposition occurs could increase with time. On the other hand, due to the brittle nature and long aspect of the nanowires, the initial length of as deposited nanowires was most probably altered by the process of freeing them from the membrane.

A mean diameter value of $60 \mathrm{~nm}$ was measured showing a $20 \%$ difference with respect to the nanopore nominal one. When investigating the homogeneity of the produced NWs, a difference was noticed at the tip - see Figure $6 \mathrm{~b}$, where the $\mathrm{ZnO} \mathrm{NW}$ starts to grow from the back-electrode. These tips showed a grainy structure that does not resemble the general structure of the NWs. The most likely explanation for the formation of this deviating structure is the formation of the double layer which requires some time. The transport of ions to and from the surface needs to stabilize and so, the initial instability would cause inhomogeneous growth that further results in a grainy structure. Also, the wire diameter is observed to be slightly varying along the nanowire length - Figure $6 \mathrm{~b}$. This is due to the so called 


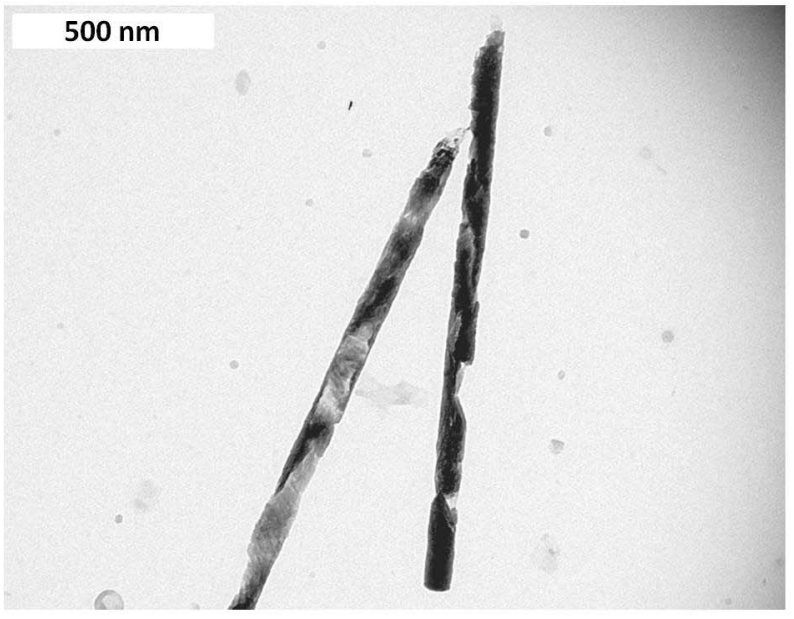

(a)

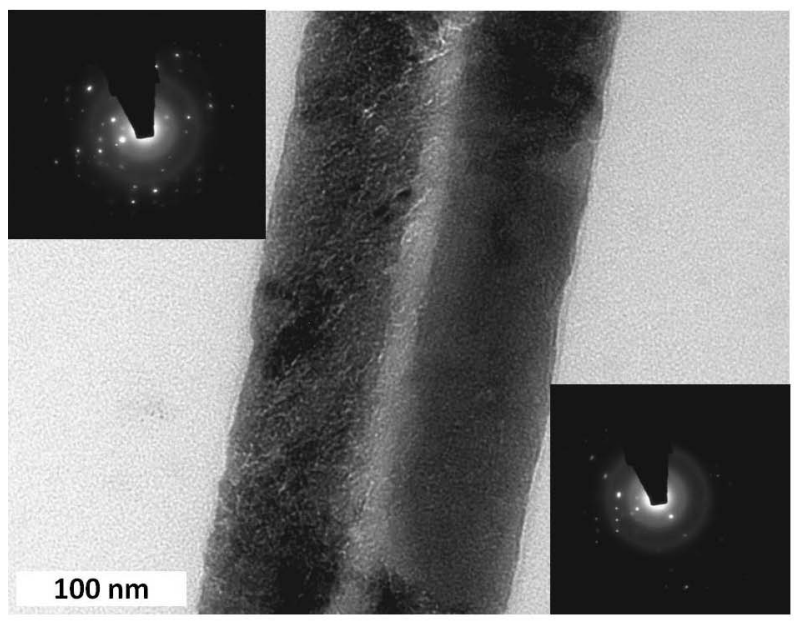

(c)

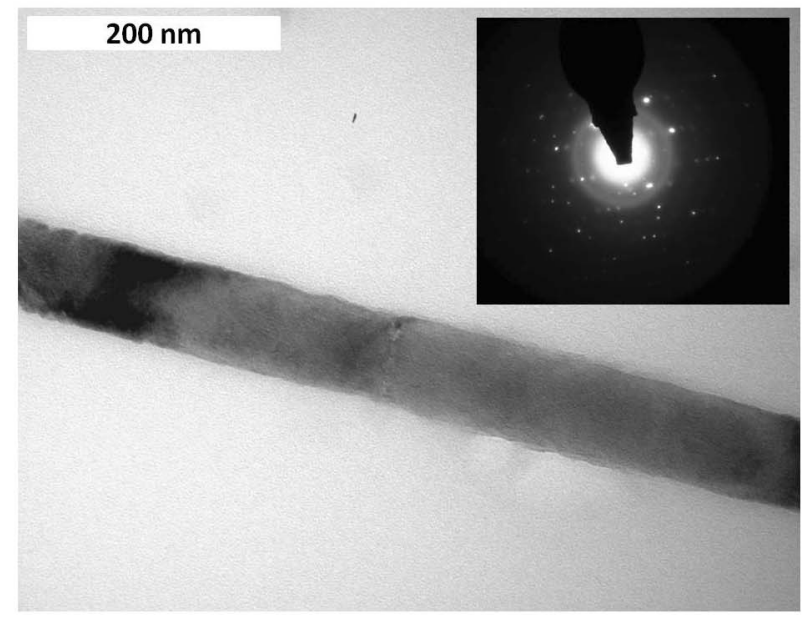

(b)

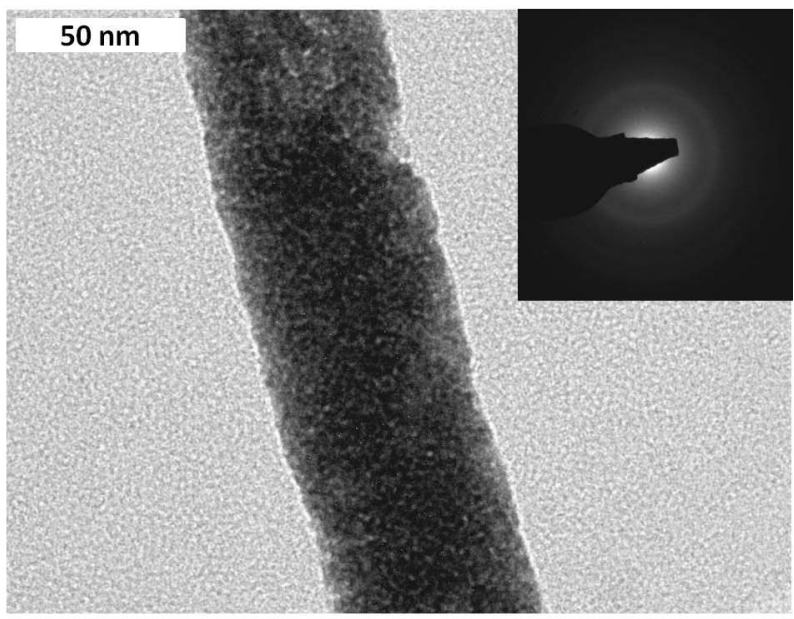

(d)

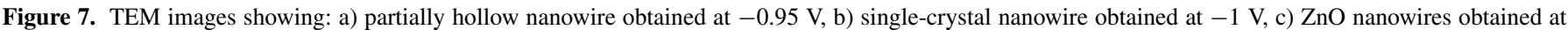
$-1.05 \mathrm{~V}$ and corresponding SAED ring patterns and d) fine grain nanostructured wire obtained at $-1.3 \mathrm{~V}$ and corresponding SAED ring patterns.

"cigar shape" of the pores that is inherent to the fabrication process (ion bombardement and dissolution) of these kind of membranes. ${ }^{36,37}$ Pseudomorphism, as referring to the continuing of grain boundaries and micro-geometrical features of the cathode substrate into the overlying deposit could be also recalled here. ${ }^{38}$

The majority of nanowires examined in Figure 6 present monocrystalline portions several microns in length. In fact, Figure $6 \mathrm{c}$ shows a single-crystal nanowire as indicated by the SAED pattern. To support the results obtained, Figure $6 \mathrm{~d}$ presents three portions of nanowires obtained in the same conditions, all with single-crystalline structure and with the same orientation as SAED pattern had the same orientation of all three. This is indicative of a common nucleation site, probably below the nanoporous template. In fact, the selection of substrate is also very important for the growth of high-quality $\mathrm{ZnO}$ nanowires, as their growth depends greatly on the lattice characteristics both of the electrodeposited material and substrate. ${ }^{39}$

In order to gain a further insight into the structure of the nanowires, different values of deposition potential were employed. Therefore, at the deposition potential of $-0.95 \mathrm{~V}$, some partially hollow nanowires were developed - see Figure 7a. This could be attributed to the lower diffusion coefficient of electroactive species and to the larger nuclei formed at more anodic potentials. By increasing the deposition potential at $-1 \mathrm{~V}$, more portions of single crystalline structure were observed - Figure 7b. In this case, the majority of nanowires consisted of both large monocrystalline areas and some small polycrystalline ones. Further increasing the deposition potential to $-1.05 \mathrm{~V}$, it can be observed that most of nanowires presented single-crystalline structure; yet some nanowires present many defects - see Figure 7c. For sufficiently small current density (or overpotential), the $\mathrm{ZnO}$ will be monocrystalline, largely replicating the substrate orientation.

By further increasing the potential to $-1.3 \mathrm{~V}$ and taking into consideration that metallic zinc is thermodynamically favorable at values more negative than $-1.3 \mathrm{~V}$, the crystal order of the nanowires seems to be lost - see Figure 7d. Anyhow, working at high solution temperature any deposited zinc metal should rapidly oxidize to $\mathrm{ZnO}$. Nevertheless, the deposition potential has a strong influence on properties of $\mathrm{ZnO}$ nanowires leading to specific structures. In fact, the nanowire structure in Figure 7d consists of fine grained crystals (nanosized) as indicated by the SAED pattern. At sufficiently large current density and/or $\mathrm{ZnO}$ thickness, a transition occured from the epitaxial single crystal to the polycrystalline structure. ${ }^{40}$ Deposition parameters determine the defect structure and texture of the deposit, above a critical deposit thickness.

The defects and polycrystalline portions present in the samples were therefore related to the influence of membrane and to the heterogeneous nucleation all along the nanopore walls. As for Peulon et al., ${ }^{18}$ other issues as membrane or substrate defects can contribute to turn a crystalline structure into an amorphous one. The roughness of the pore 
walls influences drastically the quality of the nanowires fabricated inside them, ${ }^{19}$ therefore explanations could be found in the etching step from the preparation of TE-PC which proves to be very critical. The solution used for the etching in the preparation of TE-PC membrane has a high selectivity for damage tracks. For polycarbonate, this selectivity is found to be close to $400 .{ }^{41}$ Besides the primary damage, secondary electrons generated by electronic collision cascade ${ }^{42}$ would interact destructively with the polymer membrane and therefore, resulting smaller polymeric fragments are etched more easily than the original polymer. Contamination and stressing effects, as well as a low ordering degree of pores could be considered as well.

Moreover, the density of nuclei is greatly affected by the deposition potential. The nuclei were the largest and with smaller population density at more anodic potentials while at more negative values of the deposition potential, a higher nuclei population density per $\mathrm{cm}^{2}$ is obtained. Since the amount of $\mathrm{ZnO}$ deposited was constant for each condition, the surface with the highest population density had to have the smallest nuclei and so, the dependence on the potential of the number of active sites is demonstrated. ${ }^{43}$

Taking into account the results presented, this work proved that by control of deposition parameters - here the deposition potential tailoring of nanowires structure (partially hollow, single crystalline, fine grained or fully amorphous structures) could be allowed.

\section{Conclusions}

In this work, single-crystal $\mathrm{ZnO}$ nanowires were successfully obtained by one-step electrodeposition in TE-PC membranes. As a proof of achievement, the dependence of their structural characteristics on the growth process is reported. Control of the aspect ratio of nanowires by changing the applied overpotential was proved. Varying the deposition potential from $-0.95 \mathrm{~V}$ to $-1.3 \mathrm{~V}$ results in a diffusion coefficient of almost one order of magnitude higher. Deposition rate of $\mathrm{ZnO}$ nanowires ranged $0.4 \mathrm{~nm} \mathrm{~s}^{-1}$ to $0.6 \mathrm{~nm} \mathrm{~s}^{-1}$. A $20 \%$ discrepancy between the nominal pore diameter and the nanowire diameter was observed. The obtained nanowires generally exhibited high crystal quality with a good integrity. The properties of the $\mathrm{ZnO}$ nanowire are directly related to the properties of the nanoporous template, such as the pore size distribution, surface roughness of the nanopores and relative pore orientations in the assembly.

The ability to obtain partially hollow nanowires, fine grained structures, or single crystal nanowires as well as fully amorphous nanoobjects by simply changing the deposition potential was demonstrated. Finally, the template electrodeposition reported in the present work is a cost-effective and very powerful method to grow and tailor long $\mathrm{ZnO}$ nanowires. This technology paves the way for the development and understanding of $\mathrm{ZnO}$ properties employed in a large variety of $\mathrm{ZnO}$ based devices such as LED and lasers, sensors, etc.

\section{Acknowledgments}

This work was supported by the European Commission through the program PEOPLE, by the project no. MRTN-CT-2006-035884.

\section{References}

1. M. H. Huang, S. Mao, H. Feick, H. Yan, Y. Wu, H. Kind, E. Weber, R. Russo, and P. D. Yang, Science, 292, 1897 (2001).

2. J. S. Jie, G. Z. Wang, X. H. Han, J. P. Fang, Q. X. Yu, Y. Liao, B. Xu, Q. T. Wang, and J. G. Hou, J. Phys. Chem. B, 108, 8249 (2004).

3. J. B. K. Law and J. T. L. Thong, Nanotechnology, 19, 205502 (2008).

4. T.-Jen Hsueh, C.-L. Hsu, S.-J. Chang, and I.-C. Chen, Sensor. Actuat. B: Chem., 126, 473 (2007).

5. T.-L. Phan, S. C. Yu, R. Vincent, N. H. Dan, and W. S. Shi, J. Lumin., 130, 1142 (2010).

6. L. Irimpan, V. P. N. Nampoori, and P. Radhakrishnan, Sci. Adv. Mat., 2, 578 (2010).

7. A. Pruna, V. Brânzoi, and F. Brânzoi, Rev. Roum. Chim., 55, 293 (2010).

8. S. Peulon and D. Lincot, Adv. Mater, 8, 166 (1996).

9. M. Popescu, R. Piticescu, E. Vasile, D. Taloi, M. Petriceanu, M. Stoiciu, and V. Badilita, Z. Naturforsch, 65b, 1024 (2010)

10. D. Pullini and D. Busquets Mataix, ACS Appl. Mater. Interfaces, 3, 759 (2011).

11. A. Pruna, V. Brânzoi, and F. Brânzoi, J. Appl. Electrochem., 41, 77 (2011).

12. V. Brânzoi, A. Pruna, and F. Brânzoi, Mol. Cryst. Liq. Cryst., 485, 853 (2008).

13. A. Pruna and L. Pilan, Comp. B: Eng. (2012) (in press).

14. S. P. Anthony, J. I. Lee, and J. K. Kim, Appl. Phys. Lett., 90, 103107 (2007)

15. D. Pullini, A. Pruna, S. Zanin, and D. Busquets Mataix, J. Electrochem. Soc., 159, E45 (2012)

16. J. Cembrero and D. Busquets-Mataix, Thin Solid Films, 517, 2859 (2009)

17. X. Y. Gan, X. D. Gao, J. J. Qiu, and X. M. Li, Appl. Surf. Sci., 254, 3839 (2008)

18. S. Peulon and D. Lincot, J. Electrochem. Soc., 145, 864 (1998).

19. Y. F. Gao and K. Koumoto, Cryst. Growth Des., 5, 1983 (2005).

20. Z. R. Tian, J. A. Voigt, J. Liu, B. Mckenzie, M. J. Mcdermott, M. A. Rodriguez, H. Konishi, and H. Xu, Nat. Mater, 2, 821 (2003).

21. Y. Leprince-Wang, A. Yacoubi-Ouslim, and G. Y. Wang, Microelectron. J., 36, 625 (2005).

22. B. Liu and H.-C. Zeng, J. Am. Chem. Soc., 126, 16744 (2004).

23. Z.-L. Wang, Mater. Today, 7, 26 (2004).

24. M. H. Huang, S. Mao, H. Feick, H. Yan, Y. Wu, H. Kind, E. Weber, R. Russo, and P. Yang, Science, 292, 1897 (2001).

25. J.-C. Puippe and F. Leaman, Theory and Practice of Pulse Plating, American Electroplaters and Surface Finishers Society, Orlando, FL (1986).

26. J. Elias, R. Tena-Zaera, and C. Lévy-Clément, J. Electroanal. Chem., 621, 171 (2008).

27. L. Heerman and A. Tarallo, J. Electroanal. Chem., 470, 70 (1999).

28. S. Valizadeh, J. M. George, P. Leisner, and L. Hultman, Electrochim. Acta, 47, 865 (2001).

29. A. J. Bard and L. R. Faulkner, Electrochemical Methods: Fundamentals and Applications, 2nd Ed., Wiley, New York (2001).

30. Southampton Electrochemical Group, Instrumental Methods in Electrochemistry, p. 26-31, 42-68, John Wiley \& Sons, New York (1985).

31. R. J. Brodd and V. E. Leger, Encyclopedia of Electrochemistry of the Elements, vol. V, in A. J. Bard Editor, p. 11, Marcel Dekker, New York (1976).

32. E. Ahlberg, F. Falkenberg, J. A. Manzanares, and D. J. Schiffrin, J. Electroanal. Chem., 548, 85 (2003)

33. B. R. Scharifker, J. Electroanal. Chem., 240, 61 (1988).

34. A. H. Muhr and J. M. Blanshard, Polymer, 23, 1012 (1982).

35. W. H. Safranek, The Properties of Electrodeposited Metals and Alloys, 2nd ed., American Electroplaters and Surface Finishers Society, Orlando, FL (1986).

36. D. Pullini, D. Busquets, A. Ruotolo, G. Innocenti, and V. Amigo, J. Magn. Magn. Mater, 316, e242 (2007)

37. D. Pullini, G. Innocenti, D. Busquets, and A. Ruotolo, Appl. Phys. Lett., 90, 133106 (2007).

38. R. Weil, Electroplating Engineering Handbook, 4th ed., Chapter 12, L. J. Durney Editor, Van Nostrand Reinhold (1984).

39. R. Ghosh, D. Basak, and S. Fujihara, J. Appl. Phys., 96, 2689 (2004)

40. J. Bebezuk de Cuminsky, J. Crystal Growth, 41, 330 (1977).

41. E. Ferain and R. Legras, Nucl. Instrum. Meth. B, 84, 331 (1994).

42. B. E. Fischer and R. Spohr, Rev. Mod. Phys., 55, 907 (1983).

43. D. Grujicic and B. Pesic, Electrochim. Acta, 47, 2901 (2002) 